\title{
High Prevalence of Metabolic Syndrome in Patients with Discoid Lupus Erythematosus: A Cross-Sectional, Case-Control Study
}

\author{
Sevgi Akarsu, Ozlem Ozbagcivan, Fatma Semiz, and Sebnem Aktan \\ Department of Dermatology, Dokuz Eylul University, Faculty of Medicine, Izmir, Turkey \\ Correspondence should be addressed to Ozlem Ozbagcivan; ozlem.ozbagcivan@deu.edu.tr
}

Received 23 September 2016; Accepted 7 December 2016; Published 3 January 2017

Academic Editor: Margarete D. Bagatini

Copyright (C) 2017 Sevgi Akarsu et al. This is an open access article distributed under the Creative Commons Attribution License, which permits unrestricted use, distribution, and reproduction in any medium, provided the original work is properly cited.

\begin{abstract}
Although it is known that systemic form of lupus erythematosus (LE) and metabolic syndrome (MetS) are frequently observed together, there are no published reports on MetS in patients with skin-restricted LE. We aimed to compare the frequencies of MetS and its components in discoid LE (DLE) with the non-DLE control group. Additionally, we intended to determine the differences of sociodemographic and clinical data of the DLE patients with MetS compared to the patients without MetS. This was a crosssectional, case-control study, including 60 patients with DLE and 82 age- and gender-matched control subjects. In DLE group, the presence of MetS was observed as more frequent (48.3\% versus $24.4 \%, p=0.003)$, and hypertriglyceridemia $(43.3 \%$ versus $22.0 \%$, $p=0.006)$ and reduced HDL-cholesterol (61.7\% versus $23.2 \%, p<0.001)$ among the MetS components were found significantly higher when compared to the control group. DLE patients with MetS were at older age $(50.45 \pm 11.49$ versus $43.06 \pm 12.09, p=$ 0.02 ), and hypertension, hyperlipidemia/dyslipidemia, and cardiovascular disease histories were observed at a higher ratio when compared to the patients without MetS. Between the DLE patients with and without MetS, no significant difference was observed in terms of clinical characteristics of DLE. Moreover, further large case-control studies with follow-up periods would be required to clearly assess the impact of MetS on the clinical outcomes of DLE.
\end{abstract}

\section{Introduction}

Lupus erythematosus (LE) is a chronic relapsing immunemediated inflammatory disease, characterized by a wide spectrum of presenting symptoms which range from a localized cutaneous form (cutaneous LE, CLE) to a life-threatening systemic form (systemic LE, SLE). CLE, defined as isolated cutaneous lupus lesions occurring in the absence of significant evidence of SLE, is two to three times more frequent than SLE. Chronic discoid LE (DLE) is the most common form of CLE. DLE occurs more frequently in women in their fourth and fifth decades of life, which appears to be well-demarcated, erythematous, scaly, keratotic plaques and eventually leading to disfiguring scarring and skin atrophy. Patients with DLE generally have a more benign disease course as compared to patients with other CLE subtypes, with only a reported 5-10\% developing SLE throughout their disease course [1-3].

DLE is a multifactorial disease that involves a complex interplay between genetics and environmental triggers in the setting of adaptive and innate immune response. The mechanism has not been fully elucidated, but increasing evidence suggests the role of ultraviolet irradiation, immune dysregulation, aberrant cell signaling pathways through the cytokine cascades (T helper-1 mediators including IFN- $\alpha$, IL-1, IL-6, IL-8, IL-10, and TNF- $\alpha$ ), apoptosis, necrosis, autoantibodies, plasmacytoid dendritic cells, T cells, B cells, and vascular changes [1-3]. Although SLE and DLE are clinically different diseases, there are multiple genetic similarities between the two groups to consider a common pathogenesis $[2,3]$. Although inflammatory load is lower in the patients with LE having only skin involvement; when pathogenesis is considered, it can be thought that some comorbidities accompanying SLE can also be seen in CLE patients. Hence, in the recent studies, it has been shown that some disorders such as cardiovascular diseases (CVD) and cerebrovascular accidents that are mostly associated with SLE are higher in CLE patients when compared to the control group [4-6]. Furthermore, it has been determined that metabolic syndrome (MetS) components, such as central obesity, hypertension (HT), dyslipidemia, and glucose intolerance that are known 
to be the risk factors in the occurrence of such diseases, are frequent in SLE patients and the presence of MetS accompanying SLE has been found to have higher ratios (varying from 17\% to 40\%) when compared to the general population [7-16]. Although the association of SLE and MetS has been known, no study examining the frequency of MetS in the patients with skin-restricted lupus is found in the literature.

In this study, we aimed to determine the existing MetS components and the presence of MetS in DLE patients and to compare their frequencies with the control group. In addition, we intended to describe the differences of DLE patients with MetS and the patients without MetS in terms of sociodemographic data, clinical findings, and disease severity scores.

\section{Materials and Methods}

2.1. Study Design. This was a cross-sectional, case-control study, including 60 patients with DLE and 82 age- and gender-matched control subjects who have consecutively admitted to the Department of Dermatology of Dokuz Eylul University Faculty of Medicine in Izmir, Turkey, between February 2015 and June 2016. The study protocol was approved by the Local Ethical Committee which follows the guidelines set by the Declaration of Helsinki. Only DLEdiagnosed patients who had not meet SLE classification criteria were enrolled in this study. Diagnosis of DLE was based on clinicopathological presentation and additional laboratory data, if necessary. The control group included subjects without any chronic inflammatory skin disease. Exclusion criteria were $<18$ years old, pregnancy, and cases who had received drugs in the past that alter the metabolic parameters such as systemic corticosteroids, retinoid acid, cyclosporine, and methotrexate. Each participant gave a written informed consent prior to the examinations. After signing the informed consent, sociodemographic findings (age, gender, and smoking habits) and medical histories including diabetes mellitus (DM) and/or antidiabetic use, HT and/or antihypertensive use, hyperlipidemia/dyslipidemia and/or antihyperlipidemic use, CVD (coronary artery disease including angina, myocardial infarction, coronary bypass and stroke, peripheral arterial disease, and heart failure), and depression and/or anxiety of all the patients and the control cases were recorded. A basic psychiatric interview was requested from the psychiatric department for patients who described depression and/or anxiety and/or antidepressant and/or anxiolytic drug use to confirm the diagnosis. But standard questionnaires were not used to determine the diagnosis and severity of depression and anxiety.

We considered that current smokers were those who were smoking at the time of the interview and had been smoking at least 4 cigarettes/day for 4 years [17]. In DLE patients, disease-specific findings such as disease duration, history of daily use of antimalarial treatment for more than 3 months, location and distribution of the lesions, and Cutaneous Lupus Erythematosus Disease Area and Severity Index (CLASI) scores were also recorded. Then, laboratory examinations and biometric measurements were performed both in DLE patients and in the control group in terms of dyslipidemia and MetS components, and the presence of MetS was investigated.

2.2. Clinical Severity Measurements in DLE Patients. In all DLE patients, severity of lesions was evaluated according to the CLASI which consists of activity and damage scores. This tool considers the anatomical location and morphology of the lesions and has been validated for use by both dermatologists and rheumatologists $[18,19]$. The activity score considers erythema (0-3), scale/hypertrophy (0-2), mucous membrane lesions (0-1), recent hair loss (0-1), and nonscarring alopecia $(0-3)$, and the damage score considers dyspigmentation (0$1)$, scarring/atrophy/panniculitis (0-2), and scarring of the scalp (0-6). Patients were asked for their dyspigmentation durations and the dyspigmentation score was doubled if it lasted for 12 months or longer [19].

2.3. Biometric and Laboratory Screenings. Body mass index (BMI) was calculated using the formula weight $(\mathrm{kg}) / \mathrm{height}$ $(\mathrm{m})^{2}$. Waist circumference $(\mathrm{cm})$ was measured along the line lying midway between the iliac crest and the costal margin in the midaxillary line. Blood pressure (BP) was obtained using the validated device at our center and recordings were made for two times with 1-minute intervals, and the average of both values was taken as the true BP of the patients. Blood samples were taken after an overnight fasting of $12 \mathrm{~h}$ and levels of glucose (fasting blood glucose, FBG), total cholesterol (TC), triglycerides (TG), high-density lipoprotein cholesterol (HDL-C), and low-density lipoprotein cholesterol (LDL-C) were measured. The presence of dyslipidemia was defined as serum TG $>150 \mathrm{mg} / \mathrm{dL}$, TC $>200 \mathrm{mg} / \mathrm{dL}$, LDLC $>130 \mathrm{mg} / \mathrm{dL}$, and/or antihyperlipidemic treatment [20]. When calculating the mean values of TC, TG, LDL-C, HDL$\mathrm{C}$, FBG, and systolic BP/diastolic BP, the values of the patients using antihyperlipidemic treatment, hypoglycemic treatment, and antihypertensive treatment were excluded from the calculation because of the fact that they will not reflect the actual values.

2.4. Assessment of MetS. The presence of MetS at study visit was assessed in line with the consensus criteria which were recently approved by Alberti et al. as a joint interim statement of the International Diabetes Federation Task Force on Epidemiology and Prevention; National Heart, Lung, and Blood Institute; American Heart Association; World Heart Federation; International Atherosclerosis Society; and International Association for the Study of Obesity [21]. Patients were accepted to have MetS if three or more of the following criteria were present: increased waist circumference $(\geq 80 \mathrm{~cm}$ for female, $\geq 94 \mathrm{~cm}$ for male), increased TG $(\geq 150 \mathrm{mg} / \mathrm{dL}$ or drug treatment for hypertriglyceridemia), reduced HDLC $(<50 \mathrm{mg} / \mathrm{dL}$ for female, $<40 \mathrm{mg} / \mathrm{dL}$ for male, or drug treatment for reduced HDL-C), arterial HT (systolic $\geq 130$ and/or diastolic $\geq 85 \mathrm{~mm} \mathrm{Hg}$ or drug treatment for HT), and increased FBG $(\geq 100 \mathrm{mg} / \mathrm{dL}$ or drug treatment for DM) $[21,22]$. 
2.5. Statistical Analyses. The statistical analyses were performed with the SPSS/PC software (Version 22.0 for Windows; SPSS Inc., Chicago, IL). Constant variables in the data set were expressed as mean \pm standard deviation, and categorical variables were expressed as frequency and percentage. The Shapiro-Wilk test was used to examine the normality of the distribution of the data. Two samples' Student's $t$-test was used to compare mean values of normally distributed quantitative variables as the two samples were obtained independently. Mann-Whitney $U$ test was used if the variables were not normally distributed. Qualitative variables were analyzed with chi-squared test and Fisher's exact test. $p<0.05$ was considered significant in all analyses.

\section{Results}

The average age of 60 DLE patients included in this ageand gender-matched controlled study was $46.63 \pm 12.28$ years old and the gender of majority was female (female/male: 2.33). In DLE group, it was determined that the presence of MetS was more frequent than the control group (48.3\% versus $24.4 \%, p=0.003)$, and, among the MetS components, only hypertriglyceridemia $(43.3 \%$ versus $22.0 \%, p=0.006)$ and reduced HDL-C $(61.7 \%$ versus $23.2 \%, p<0.001)$ were found to be significantly higher. Additionally, being current smokers, depression/anxiety history, and presence of dyslipidemia were significantly higher in DLE patients than the control group (Table 1). Five (8.3\%) of DLE patients $(2(3.3 \%)$ had angina, $2(3.3 \%)$ had myocardial infarction, and $1(1.7 \%)$ had coronary bypass) and $4(4.9 \%)$ of controls (3 (3.6\%) had angina and $1(1.2 \%)$ had myocardial infarction) had coronary artery disease in means of CVD.

When DLE patients with and without MetS were compared with each other, it was determined that the patients with MetS did not show any difference in terms of gender distribution when compared to the patients without MetS; however they were at older age $(50.45 \pm 11.49$ versus $43.06 \pm$ $12.09, p=0.02$ ). In addition, while no statistically significant difference was found between both groups in terms of smoking status and mean BMI values, HT and/or antihypertensive use, hyperlipidemia/dyslipidemia and/or antihyperlipidemic use, and CVD histories were found higher in DLE patients with MetS. In DLE patients with MetS, the frequencies of MetS components were found to have increased waist circumference $(86.2 \%)$, reduced HDL-C (79.3\%), hypertriglyceridemia (72.4\%), elevated BP (62.1\%), and increased FBG (58.6\%), respectively. As expected, all individual components of MetS were more common in patients with MetS than in those without (Table 2).

It was determined that the prevalence of CVD was increasing by age in a statistical significance in DLE patients, as well as the prevalence of MetS $(p=0.001)$. DLE patients with MetS who had CVD had statistically significant higher mean age $(64.00 \pm 9.46)$ compared to those without CVD $(45.00 \pm 11.29)(p=0.002)$. In partial correlation analysis, ignoring the age factor, the association between MetS and CVD did not reach a statistical significance $(p=0.109)$.
No statistically significant difference was observed between the DLE patients with and without MetS in terms of DLE disease duration, use of antimalarial treatment, lesion locations, distribution of lesions, and the mean total CLASI activity score, damage score, and subscale scores (Table 3).

\section{Discussion}

In this study, we have determined the prevalence of MetS and its individual components in a sample of DLE patients from Turkey. As a result, we found higher rates of MetS in DLE patients (48.3\%) than the control group as well as lower values of HDL-C (61.7\%) and higher values of triglycerides (43.3\%) among the MetS components. In DLE patients with MetS, histories of hyperlipidemia/dyslipidemia, HT, and CVD were notified more frequent, and the presence of dyslipidemia and all MetS components was observed at a significantly higher ratio than the patients without MetS through the investigations.

MetS is characterized by a group of interrelated metabolic risk factors, including the central obesity, atherogenic dyslipidemia, elevated BP, and increased FBG. It is known that these risk factors promote the development of atherosclerotic CVD and/or type $2 \mathrm{DM}$. The prevalence of MetS has increased in the last few years throughout the world. Approximately, one-third of the adult population in industrialized and also developing countries can be categorized as having MetS by different definitions $[22,23]$. Recent evidences suggest that SLE has been associated with MetS as well as its various components [7-16]. The most important reason of the longterm mortality in SLE is premature atherosclerosis and CVD and, in such patients, it has been observed that both MetS and MetS components have higher prevalence and also cumulative organ damage correlated with SLE in the presence of MetS is higher [10-15]. Some authors have mentioned that whether lupus activity is a factor in the development of MetS or whether MetS is contributing to disease activity is unclear. Inflammation is not only a triggering factor of the MetS but also a consequence. It is thought that increased circulating adipocytokines such as TNF- $\alpha$, IL-6, leptin, resistin, plasminogen activator inhibitor-1, and acute-phase reactants such as C-reactive protein could have an important role in the development of MetS and/or its components and its association with disease activity in SLE patients [12-15].

As known, a critical and most important part of assessment of DLE patients is evaluating and monitoring for early detection of SLE development [1-3]. Furthermore, especially in recent years, evaluating the significant accompanying comorbidities in terms of morbidity and mortality such as CVDs and cerebrovascular accidents (which are more frequent in SLE) has been highlighted in skin-restricted lupus patients [4-6]. However, it is unknown that whether CLE is associated with risk of MetS or not. To date, there are no published reports on MetS and associated factors in patients with CLE.

Recently, in a large case-control study performed by Hesselvig et al. in Danish population, significantly increased risk of CVDs and all-cause mortality were observed both in 
TABLE 1: Comparison of sociodemographic features, medical history findings, and metabolic syndrome components in study subjects.

\begin{tabular}{|c|c|c|c|}
\hline Characteristics & $\operatorname{DLE}(n=60)$ & Controls $(n=82)$ & $p$ value \\
\hline Age, years, mean \pm SD & $46.63 \pm 12.28$ & $46.40 \pm 12.00$ & 0.911 \\
\hline \multicolumn{4}{|l|}{ Gender, $n(\%)$} \\
\hline Male & $18(30)$ & $25(30.5)$ & 0.950 \\
\hline Female & $42(70)$ & $57(69.5)$ & \\
\hline Current smokers, $n(\%)$ & $34(56.7)$ & $26(31.7)$ & $\mathbf{0 . 0 0 3}^{*}$ \\
\hline Packet/year & $16.53 \pm 14.73$ & $4.02 \pm 8.26$ & $<0.001^{*}$ \\
\hline \multicolumn{4}{|l|}{ Medical history, $n(\%)$} \\
\hline Diabetes mellitus and/or antidiabetic use & $6(10)$ & $9(11)$ & 0.852 \\
\hline Hypertension and/or antihypertensive use & $18(30)$ & $14(17.1)$ & 0.069 \\
\hline Hyperlipidemia/dyslipidemia and/or antihyperlipidemic use & $4(6.7)$ & $1(1.2)$ & 0.162 \\
\hline Cardiovascular disease & $5(8.3)$ & $4(4.9)$ & 0.494 \\
\hline Depression and/or anxiety & $24(40)$ & $7(8.5)$ & $<0.001^{*}$ \\
\hline Body mass index, mean \pm SD & $27.08 \pm 3.90$ & $26.67 \pm 4.95$ & 0.385 \\
\hline Dyslipidemia (\%) & $40(66.7)$ & $38(46.3)$ & $0.016^{*}$ \\
\hline Metabolic syndrome & $29(48.3)$ & $20(24.4)$ & $0.003^{*}$ \\
\hline Increased waist circumference $\geq 94 \mathrm{~cm}$ (male) or $\geq 80 \mathrm{~cm}$ (female) & $38(63.3)$ & $49(59.8)$ & 0.666 \\
\hline Hypertriglyceridemia $\geq 150 \mathrm{mg} / \mathrm{dL}$ or antihyperlipidemic treatment & $26(43.3)$ & $18(22.0)$ & $0.006^{*}$ \\
\hline Reduced HDL-cholesterol < 50 mg/dL (female) or < 40 mg/dL (male) & $37(61.7)$ & $19(23.2)$ & $<0.001^{*}$ \\
\hline Elevated blood pressure $\geq 130 / 85 \mathrm{mmHg}$ or antihypertensive treatment & $25(41.7)$ & $26(31.7)$ & 0.222 \\
\hline Increased fasting blood glucose $\geq 100 \mathrm{mg} / \mathrm{dL}$ or hypoglycemic treatment & $20(33.3)$ & $22(26.8)$ & 0.402 \\
\hline Number of metabolic syndrome components & $2.37 \pm 1.33$ & $1.48 \pm 1.27$ & $<0.001^{*}$ \\
\hline
\end{tabular}

${ }^{*}$ Statistically significant values.

TABLE 2: Comparison of sociodemographic features, medical history findings, and metabolic syndrome components in DLE patients with and without metabolic syndrome.

\begin{tabular}{|c|c|c|c|}
\hline Characteristics, $n(\%)$ & $\begin{array}{c}\text { DLE patients } \\
\text { with MetS } \\
(n=29)\end{array}$ & $\begin{array}{l}\text { DLE patients } \\
\text { without MetS } \\
\quad(n=31)\end{array}$ & $p$ value \\
\hline Age, years, mean $\pm S D$ & $50.45 \pm 11.49$ & $43.06 \pm 12.09$ & $0.020^{*}$ \\
\hline \multicolumn{4}{|l|}{ Gender, $n(\%)$} \\
\hline Male & $11(37.9)$ & $7(22.6)$ & 0.195 \\
\hline Female & $18(62.1)$ & $24(77.4)$ & \\
\hline Current smokers, $n(\%)$ & $17(58.6)$ & $17(54.8)$ & 0.768 \\
\hline Packet/year & $16.86 \pm 17.10$ & $16.22 \pm 12.42$ & 0.715 \\
\hline \multicolumn{4}{|l|}{ Medical history, $n(\%)$} \\
\hline Diabetes mellitus and/or antidiabetic use & $5(17.2)$ & $1(3.2)$ & 0.098 \\
\hline Hypertension and/or antihypertensive use & $14(48.3)$ & $4(12.9)$ & $0.003^{*}$ \\
\hline Hyperlipidemia/dyslipidemia and/or antihyperlipidemic use & $4(13.8)$ & 0 & $0.049^{*}$ \\
\hline Cardiovascular disease & $5(17.2)$ & 0 & $0.022^{*}$ \\
\hline Depression and/or anxiety & $12(41.4)$ & $12(38.7)$ & 0.833 \\
\hline Body mass index, mean \pm SD & $28.01 \pm 3.74$ & $26.22 \pm 391$ & 0.096 \\
\hline Dyslipidemia, $n(\%)$ & $23(79.3)$ & $17(54.8)$ & $0.044^{*}$ \\
\hline \multicolumn{4}{|l|}{ Metabolic syndrome components } \\
\hline Increased waist circumference $\geq 94 \mathrm{~cm}$ (male) or $\geq 80 \mathrm{~cm}$ (female) & $25(86.2)$ & $13(41.9)$ & $<0.001^{*}$ \\
\hline Hypertriglyceridemia $\geq 150 \mathrm{mg} / \mathrm{dL}$ or antihyperlipidemic treatment & $21(72.4)$ & $5(16.1)$ & $<0.001^{*}$ \\
\hline Reduced HDL-cholesterol $<50 \mathrm{mg} / \mathrm{dL}$ (female) or $<40 \mathrm{mg} / \mathrm{dL}$ (male) & $23(79.3)$ & $14(45.2)$ & $0.007^{*}$ \\
\hline Elevated blood pressure $\geq 130 / 85 \mathrm{mmHg}$ or antihypertensive treatment & $18(62.1)$ & $7(22.6)$ & $0.002^{*}$ \\
\hline Increased fasting blood glucose $\geq 100 \mathrm{mg} / \mathrm{dL}$ or hypoglycemic treatment & $17(58.6)$ & $3(9.7)$ & $<0.001$ \\
\hline
\end{tabular}

${ }^{*}$ Statistically significant values. 
TABLE 3: Comparison of clinical characteristics in DLE patients with and without metabolic syndrome.

\begin{tabular}{|c|c|c|c|}
\hline Characteristics, $n(\%)$ & $\begin{array}{l}\text { DLE patients with MetS } \\
(n=29)\end{array}$ & $\begin{array}{l}\text { DLE patients without MetS } \\
\qquad(n=31)\end{array}$ & $p$ value \\
\hline Disease duration (month), mean $\pm \mathrm{SD}$ & $74.10 \pm 61.05$ & $68.52 \pm 75.36$ & 0.210 \\
\hline Use of antimalarials & $8(27.6 \%)$ & $9(29)$ & 0.901 \\
\hline \multicolumn{4}{|l|}{ Location of DLE lesions, $n(\%)$} \\
\hline Face & $25(86.2)$ & $27(87.1)$ & 1.00 \\
\hline Scalp & $12(41.4)$ & $11(35.5)$ & 0.639 \\
\hline Trunk & $9(31)$ & $8(25.8)$ & 0.653 \\
\hline Upper extremity & $8(27.6)$ & $9(29)$ & 0.901 \\
\hline Lower extremity & $2(6.9)$ & $2(6.5)$ & 1.00 \\
\hline \multicolumn{4}{|l|}{ Distribution of DLE lesions, $n(\%)$} \\
\hline Localized & $18(62.1)$ & $21(67.7)$ & 0.645 \\
\hline Generalized & $11(37.9)$ & $10(32.3)$ & 0.645 \\
\hline \multicolumn{4}{|l|}{ CLASI scores, mean \pm SD } \\
\hline Total CLASI activity score & $9.10 \pm 9.03$ & $7.32 \pm 6.42$ & 0.588 \\
\hline Erythema & $6.00 \pm 6.24$ & $4.58 \pm 4.23$ & 0.346 \\
\hline Squama & $2.34 \pm 3.40$ & $1.90 \pm 2.20$ & 0.939 \\
\hline Mucosal involvement & $0.21 \pm 0.41$ & $0.23 \pm 0.42$ & 0.860 \\
\hline Noncicatricial alopecia & $0.76 \pm 0.99$ & $0.71 \pm 1.01$ & 0.777 \\
\hline Total CLASI damage score & $5.93 \pm 6.22$ & $4.55 \pm 5.36$ & 0.292 \\
\hline Dyspigmentation & $2.28 \pm 2.53$ & $1.58 \pm 2.25$ & 0.145 \\
\hline Scarring & $1.79 \pm 2.74$ & $1.74 \pm 1.97$ & 0.562 \\
\hline Cicatricial alopecia & $1.86 \pm 2.34$ & $1.26 \pm 1.82$ & 0.430 \\
\hline
\end{tabular}

CLE and in SLE patients including inpatients and outpatients, as being higher in SLE, and these risks were found more significant in the patients with an age of $\leq 50$ years [4]. In a Swedish study, Zöller et al. examined whether there was an association between hospital admission for CLE and subsequent risk of hospitalization for coronary heart disease. They found that the risk was at a greatest level 1-5 years after being hospitalized with CLE and age of $>50$ years with a higher standardized incidence ratio for subsequent coronary heart disease of patients with DLE after one year of follow-up [5]. Furthermore, in a population-based inception cohort of patients with CLE, while the cardiovascular death, ischemic heart disease, and heart failure risks were similar to the control group, cerebrovascular accidents and peripheral arterial disease risks were higher at a ratio of at least two times. Although these risks of cardiovascular events and cerebrovascular accidents did not show a statistical significance in generalized DLE, it was specified that such risks were higher than the patients with localized involvement; thus these comorbidities could increase depending on the extent of cutaneous involvement [6]. In our study, the presence of CVD history did not show significance in DLE patients when compared to the control group; however, it was observed higher in DLE patients with MetS when compared to the patients without MetS. Moreover, because of the absence of a significant relationship between CVD and MetS when the age factor was ignored, it can be said that the combination of CVD and MetS increases by age in DLE patients. The MetS is not an absolute risk indicator because it does not contain many of the factors that determine absolute risk, for example, age, sex, cigarette smoking, and LDLC levels. Nonetheless, patients with the MetS are at twice the risk of developing CVD over the next 5 to 10 years as individuals without the syndrome. The risk over a lifetime undoubtedly is even higher [9-13, 20]. Similarly, the ratio of generalized lesion distribution, the mean total CLASI activity, and damage scores were higher in our DLE patients with MetS than in those without MetS, but such values did not reach a statistical significance. The notion that antimalarials are protective against MetS, CVD, and general mortality in SLE patients is endorsed by some authors [13, 15], but not by others $[6,14]$. Singh et al. found that there was no difference in total cholesterol between CLE patients who had or never used antimalarials [6]. Additionally, the use of antimalarial therapy in DLE patients did not show an association with MetS in our study. Thus, more long-term results from prospective large cohorts would be needed to address these arguments.

The pathogenesis of CLE is similar to SLE, with complex gene-environment interactions, autoimmunity, and immune-mediated cutaneous damage, and, hence, conceivably, patients with CLE may have increased risk of MetS. Local and chronic inflammation in DLE patients may be the driver of low-grade systemic inflammation [2-4]. Thus, this inflammation may have thought of to be the connecting link between DLE and MetS. Although the limited extent of organ involvement with absence of systemic damage suggests that chronic systemic inflammation may be absent in 
patients with CLE, other limited autoimmune dermatological conditions such as psoriasis, alopecia, and lichen planus have also been variably associated with an increased MetS and/or its components [24].

In this study, the most frequently determined MetS components in DLE patients were increased waist circumference, reduced HDL-C, and hypertriglyceridemia conforming to the previous findings of SLE patients with MetS. Unsurprisingly, it was an expected result to find lower ratio of arterial HT in DLE patients with MetS when compared to the SLE patients with MetS. In the previous studies, older age in SLE patients has been notified with MetS similar to our DLE patients; however, different from our study, as can be interpreted as higher inflammation burden in SLE, higher $\mathrm{BMI}$, longer disease duration, and higher disease activity have been found in SLE patients with MetS when compared to the patients without MetS $[13,14]$.

Several studies have yielded crucial information implicating visceral adipose tissue as a main focus of the chronic inflammation that can contribute to MetS and its components. It has been demonstrated that the downregulation of T-regulatory cells seems to play an important role in the development of CLE and these T-regulatory cells also ameliorate the glucose intolerance in the context of central obesity. Additionally, it has been shown that B cells exacerbate glucose intolerance through the production of pathogenic immunoglobulins presumably against adipose tissue antigens [16]. In our study, the presence of increased FBG in DLE patients did not show any significance when compared to the control group, and it can be attributed to the comparable values of the indicators of visceral adipose tissue such as mean BMI and mean waist circumference in patients and control group. Also, both increased waist circumference and increased FBG frequencies were higher in the DLE patients with MetS when compared to the DLE patients without MetS.

DLE has been linked to smoking in several case-control studies, which have shown to have consistently higher smoking rates $(50-87 \%)$ in DLE patients $[17,25,26]$. It has been shown that cigarette smoking increases inflammatory cytokines, apoptosis, certain autoantibodies, the development of free radicals, and photosensitivity [17]. We found a statistically higher number of smokers among DLE patients than among controls and the number of cigarettes smoked daily was higher in patients with DLE, as found in other casecontrol reports. In previous studies, it has been determined by Negrón et al. that smoking status in SLE patients with MetS did not show any significance when compared to the control group while Mederios et al. reported that SLE patients with MetS were higher smokers when compared to the patients without MetS $[12,13]$. In our study, no significance was observed in terms of smoking habits between the DLE patients with and without MetS.

In recent years, the common association of skin-restricted LE with psychiatric disease and daily stress has been demonstrated in a few studies [27-30]. In a case-control study conducted by Jalenques et al., it was found that the most frequently observed current psychiatric disorders were major depressive disease (39\%) and anxiety disorders (35\%) in patients with skin-restricted lupus [28]. In another crosssectional study, $34.7 \%$ of the patients with DLE met the criteria for depression and/or anxiety with need of mental health care or psychotropic treatment [29]. Skin atrophy, scarring, and scarring alopecia may cause mood and anxiety disorders and can seriously impact the quality of life in patients with DLE. Additionally, anxiety and emotional factors can also precipitate DLE lesions and induce treatment resistance [2830]. It has been suggested that chronic inflammation may be a key feature linking DLE with depression and/or anxiety. Also it has been notified that the presence of depression can be related to both SLE and MetS and/or its components via common pathogenesis. In other words, depression is a contributing factor to increased (neuro)inflammatory burden; therefore it may increase the inflammatory and degenerative progression. It is now evident that depression is the clinical expression of peripheral cell-mediated activation, inflammation (increased levels of proinflammatory cytokines, such as IL- 6 and TNF- $\alpha$ ), and induction of oxidative and nitrosative stress pathways [30]. In our study, depression and/or anxiety ratio was significantly found to be higher in DLE patients when compared to the control group; however, although it was partially higher in DLE patients with MetS when compared to the patients without MetS, no statistically significance was observed.

Consequently, when the MetS frequency and the number of determined MetS components in DLE patients were compared to the non-DLE control group, they were found to be approximately two times higher. All the components in DLE patients with MetS were more frequent than in the patients without MetS and they were central obesity, reduced HDL-C, hypertriglyceridemia, HT, and glucose intolerance, respectively. In these patients, CVD history was also determined more frequently. Due to the absence of data in the literature related to the presence of MetS in skin-restricted LE patients, these results obtained from our study can be a fore step for further studies. Nevertheless, the present study has some limitations such as being a cross-sectional design and including small number of patients with DLE. Additionally, other factors known to be associated with MetS, particularly alcohol drinking, dietary and exercise habits, and proinflammatory cytokines or procoagulant factors such as TNF- $\alpha$, IL-6, and fibrinogen, were not evaluated in this study.

\section{Conclusions}

In conclusion, the frequencies of MetS and especially the MetS components correlated with atherogenic dyslipidemia, which are independent risk factors for atherosclerotic CVD, were found to be at a high ratio in DLE. Thus, it may be said that it is important to identify patients at risk to develop MetS early in the course of DLE. In such patients, in terms of reducing the risk of MetS, applying lifestyle changing strategies such as nutritional counselling and weight reduction can be recommended. Because of its design, our study does not indicate cause and effect between DLE and MetS and its components, but inflammation might be an area with potential for further investigation. Therefore, further 
large case-control studies with follow-up periods would be required to clearly assess the impact of MetS on the clinical outcomes of DLE.

\section{Competing Interests}

The authors declare that there is no conflict of interests regarding the publication of this paper.

\section{References}

[1] E. Z. Hejazi and V. P. Werth, "Cutaneous lupus erythematosus: an update on pathogenesis, diagnosis and treatment," American Journal of Clinical Dermatology, vol. 17, no. 2, pp. 135-146, 2016.

[2] V. P. Werth, "Cutaneous lupus: insights into pathogenesis and disease classification," Bulletin of the NYU Hospital for Joint Diseases, vol. 65, no. 3, pp. 200-204, 2007.

[3] M. G. Kirchhof and J. P. Dutz, "The immunopathology of cutaneous lupus erythematosus," Rheumatic Disease Clinics of North America, vol. 40, no. 3, pp. 455-474, 2014.

[4] J. H. Hesselvig, O. Ahlehoff, L. Dreyer, G. Gislason, and K. Kofoed, "Cutaneous lupus erythematosus and systemic lupus erythematosus are associated with clinically significant cardiovascular risk: a Danish nationwide cohort study," Lupus, vol. 26, no. 1, pp. 48-53, 2017.

[5] B. Zöller, X. Li, J. Sundquist, and K. Sundquist, "Risk of subsequent coronary heart disease in patients hospitalized for immune-mediated diseases: a nationwide follow-up study from Sweden," PLoS ONE, vol. 7, no. 3, Article ID e33442, 2012.

[6] A. G. Singh, C. S. Crowson, S. Singh et al., "Risk of cerebrovascular accidents and ischemic heart disease in cutaneous lupus erythematosus: a population-based cohort study," Arthritis Care \& Research, vol. 68, no. 11, pp. 1664-1670, 2016.

[7] J. M. Sabio, M. Zamora-Pasadas, J. Jiménez-Jáimez et al., "Metabolic syndrome in patients with systemic lupus erythematosus from Southern Spain," Lupus, vol. 17, no. 9, pp. 849-859, 2008.

[8] B. Parker, Y. Ahmad, J. Shelmerdine et al., "An analysis of the metabolic syndrome phenotype in systemic lupus erythematosus," Lupus, vol. 20, no. 14, pp. 1459-1465, 2011.

[9] C. P. Chung, I. Avalos, A. Oeser et al., "High prevalence of the metabolic syndrome in patients with systemic lupus erythematosus: association with disease characteristics and cardiovascular risk factors," Annals of the Rheumatic Diseases, vol. 66, no. 2, pp. 208-214, 2007.

[10] I. S. Nascimento, C. R. D. C. Quaio, N. A. Sinicato, S. Appenzeller, and J. F. De Carvalho, "Aspects of atherosclerosis and metabolic syndrome in lupus erythematosus," Acta Reumatologica Portuguesa, vol. 35, no. 3, pp. 294-300, 2010.

[11] C. C. Mok, W. L. Poon, J. P. S. Lai et al., "Metabolic syndrome, endothelial injury, and subclinical atherosclerosis in patients with systemic lupus erythematosus," Scandinavian Journal of Rheumatology, vol. 39, no. 1, pp. 42-49, 2010.

[12] A. M. Negrón, M. J. Molina, A. M. Mayor, V. E. Rodríguez, and L. M. Vilá, "Factors associated with metabolic syndrome in patients with systemic lupus erythematosus from Puerto Rico," Lupus, vol. 17, no. 4, pp. 348-354, 2008.

[13] M. M. D. C. Medeiros, Í. M. Xavier de Oliveira, and Á. T. M. Ribeiro, "Prevalence of metabolic syndrome in a cohort of systemic lupus erythematosus patients from Northeastern
Brazil: association with disease activity, nephritis, smoking, and age," Rheumatology International, vol. 36, no. 1, pp. 117-124, 2016.

[14] R. Castejon, C. Jimenez-Ortiz, S. Rosado, P. Tutor-Ureta, S. Mellor-Pita, and M. Yebra-Bango, "Metabolic syndrome is associated with decreased circulating endothelial progenitor cells and increased arterial stiffness in systemic lupus erythematosus," Lupus, vol. 25, no. 2, pp. 129-136, 2016.

[15] S. Demir, B. Artim-Esen, Y. Şahinkaya et al., "Metabolic syndrome is not only a risk factor for cardiovascular diseases in systemic lupus erythematosus but is also associated with cumulative organ damage: a cross-sectional analysis of 311 patients," Lupus, vol. 25, no. 2, pp. 177-184, 2016.

[16] C. L. Gabriel, P. B. Smith, Y. V. Mendez-Fernandez, A. J. Wilhelm, A. M. Ye, and A. S. Major, "Autoimmune-mediated glucose intolerance in a mouse model of systemic lupus erythematosus," American Journal of Physiology-Endocrinology and Metabolism, vol. 303, no. 11, pp. E1313-E1324, 2012.

[17] H. A. Miot, L. D. B. Miot, and G. R. Haddad, "Association between discoid lupus erythematosus and cigarette smoking: a case-control study," Dermatology, vol. 211, no. 2, pp. 118-122, 2005.

[18] J. Albrecht, L. Taylor, J. A. Berlin et al., "The CLASI (Cutaneous Lupus Erythematosus Disease Area and Severity Index): an outcome instrument for cutaneous lupus erythematosus," Journal of Investigative Dermatology, vol. 125, no. 5, pp. 889-894, 2005.

[19] D. Bein, E. Kuehn, A. M. Meuth et al., "Evaluation of disease activity and damage in different subtypes of cutaneous lupus erythematosus using the CLASI," Journal of the European Academy of Dermatology and Venereology, vol. 25, no. 6, pp. 652-659, 2011.

[20] Expert Panel on Detection, Evaluation, and Treatment of High Blood Cholesterol in Adults, "Executive summary of the third report of the National Cholesterol Education Program (NCEP) expert panel on detection, evaluation, and treatment of high blood cholesterol in adults (adult treatment panel III)," Journal of the American Medical Association, vol. 285, no. 19, pp. 24862497, 2001.

[21] K. G. Alberti, R. H. Eckel, S. M. Grundy et al., "Harmonizing the metabolic syndrome: a joint interim statement of the international diabetes federation task force on epidemiology and prevention; National Heart, Lung, and Blood Institute; American Heart Association; World Heart Federation; International Atherosclerosis Society; And International Association for the Study of obesity," Circulation, vol. 120, no. 16, pp. 1640$1645,2009$.

[22] R. H. Eckel, K. G. M. M. Alberti, S. M. Grundy, and P. Z. Zimmet, "The metabolic syndrome," The Lancet, vol. 375, no. 9710, pp. 181-183, 2010.

[23] P. W. F. Wilson, R. B. D’Agostino, H. Parise, L. Sullivan, and J. B. Meigs, "Metabolic syndrome as a precursor of cardiovascular disease and type 2 diabetes mellitus," Circulation, vol. 112, no. 20, pp. 3066-3072, 2005.

[24] T. Padhi and Garima, "Metabolic syndrome and skin: psoriasis and beyond," Indian Journal of Dermatology, vol. 58, no. 4, pp. 299-305, 2013.

[25] E. W. Piette, K. P. Foering, A. Y. Chang et al., "Impact of smoking in cutaneous lupus erythematosus," Archives of Dermatology, vol. 148, no. 3, pp. 317-322, 2012.

[26] B. C. Böckle and N. T. Sepp, "Smoking is highly associated with discoid lupus erythematosus and lupus erythematosus tumidus: analysis of 405 patients," Lupus, vol. 24, no. 7, pp. 669-674, 2015. 
[27] A. P. Bewley, "Cutaneous lupus erythematosus is strongly associated with psychiatric disease," British Journal of Dermatology, vol. 174, no. 5, pp. 958-959, 2016.

[28] I. Jalenques, F. Rondepierre, C. Massoubre et al., "High prevalence of psychiatric disorders in patients with skin-restricted lupus: a case-control study," British Journal of Dermatology, vol. 174, no. 5, pp. 1051-1060, 2016.

[29] J. Achtman, M. A. Kling, R. Feng, J. Okawa, and V. P. Werth, "A cross-sectional study of untreated depression and anxiety in cutaneous lupus erythematosus and dermatomyositis," Journal of the American Academy of Dermatology, vol. 74, no. 2, pp. 377379, 2016.

[30] M. Maes, M. Kubera, E. Obuchowiczwa, L. Goehler, and J. Brzeszcz, "Depression's multiple comorbidities explained by (neuro)inflammatory and oxidative \& nitrosative stress pathways," Neuroendocrinology Letters, vol. 32, no. 1, pp. 7-24, 2011. 


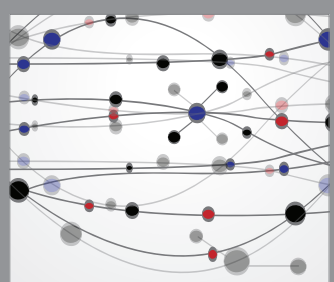

The Scientific World Journal
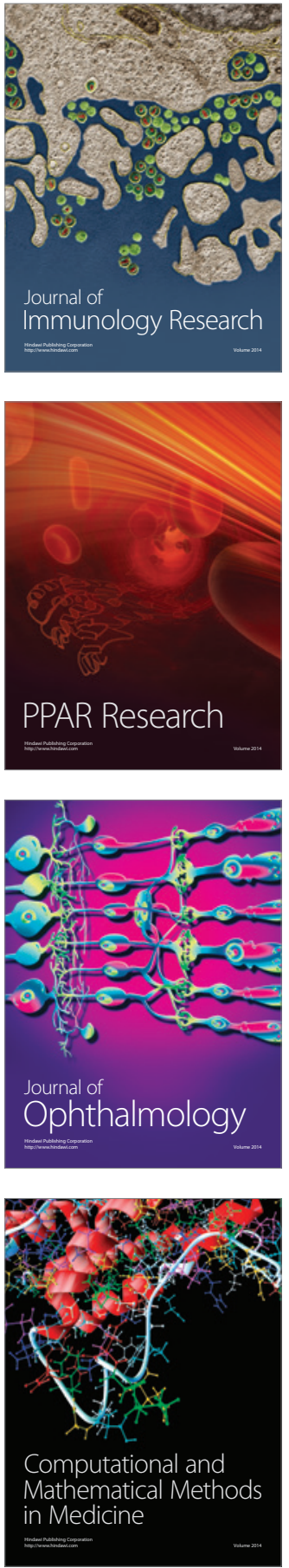

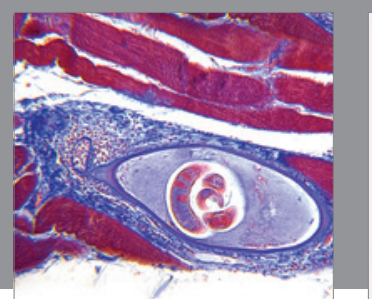

Gastroenterology Research and Practice
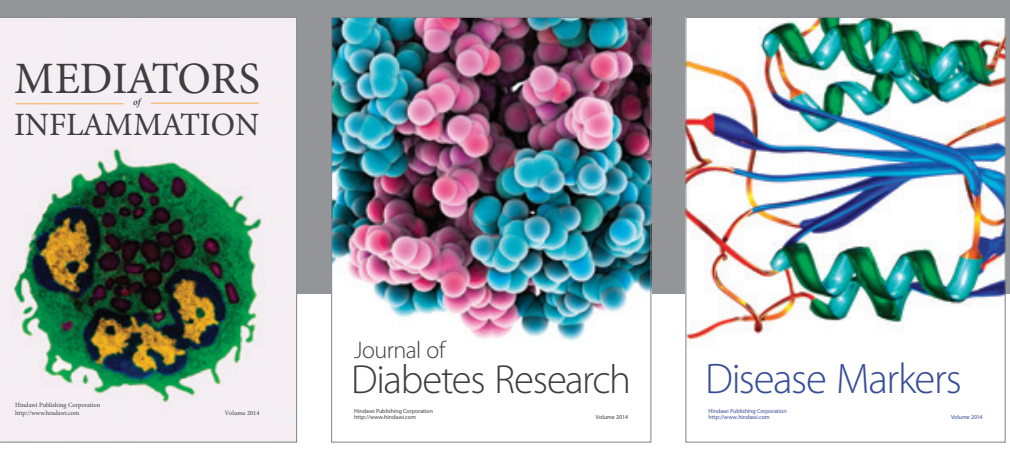

Disease Markers

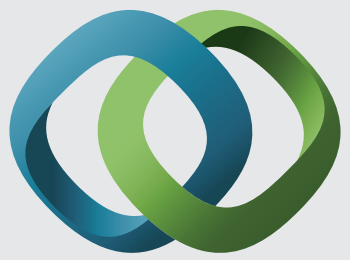

\section{Hindawi}

Submit your manuscripts at

https://www.hindawi.com
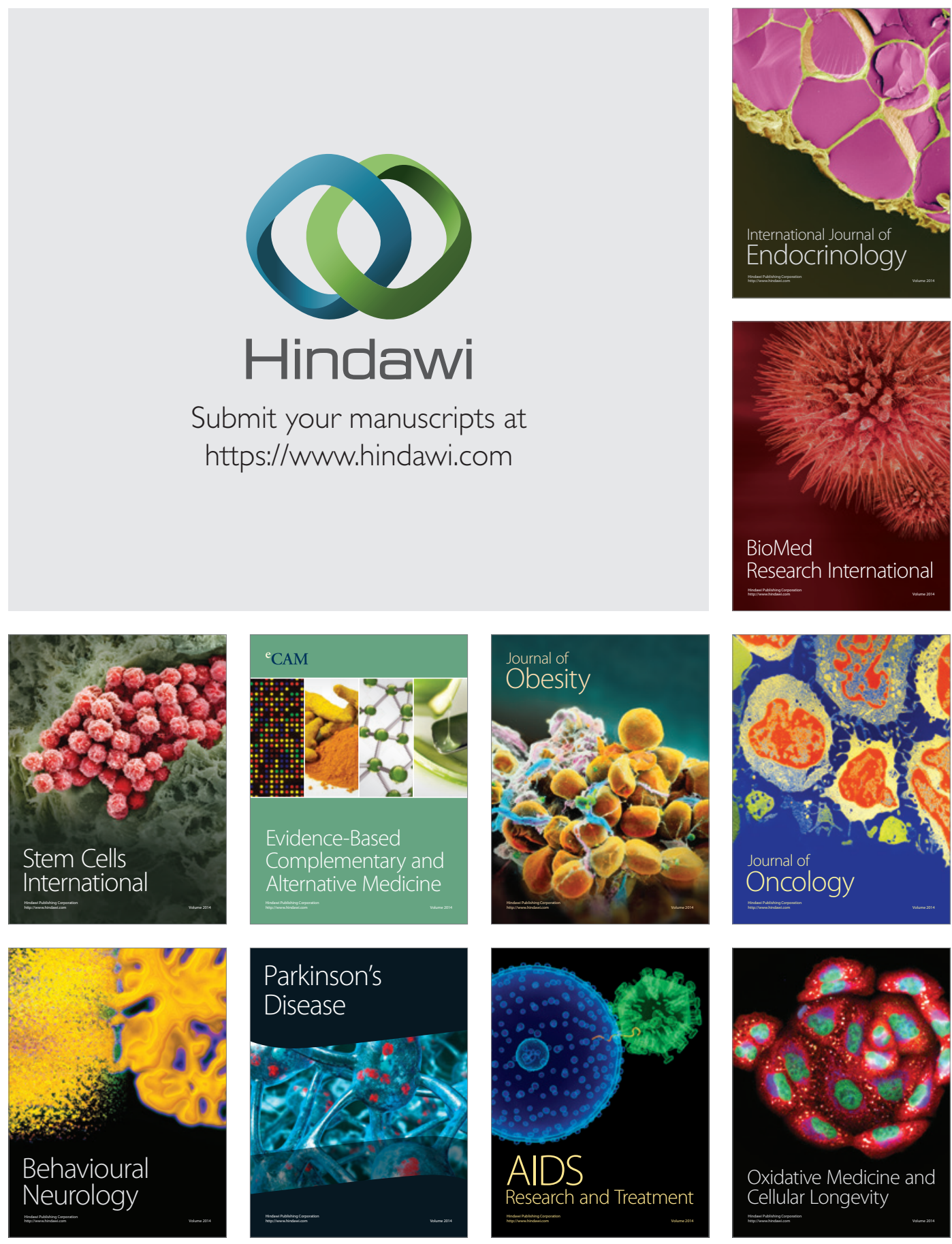